\title{
La Caldera de Coli y su vecina Guadalajara
}

\author{
Phil C. Weigand*
}

EL COLEGIO DE MICHOACÁN

Los cerros de Coli están localizados en las afueras de la ciudad de Guadalajara. Popularmente, el lugar es también conocido como La Primavera. El Coli es una caldera volcánica activa. Su formación original está fechada en la época del Pleistoceno tardío, y hoy representa un peligro para su vecina Guadalajara. Las calderas, como tipos de volcanes, son mucho más peligrosas que estratovolcanes, como lo son los volcanes de Colima y el Ceboruco. Las calderas explotan de manera violenta con muy poco aviso. Los avisos muchas veces son sutiles, pero sin el equipo para medir y vigilar las actividades geológicas en el interior de la Caldera de Coli la ciudad de Guadalajara está expuesto a un peligro innecesario. Este breve estudio representa una descripción de la formación y carácter histórico y actual de la Caldera de Coli.

(Volcanismo, Guadalajara, planeación urbana, geología)

\section{INTRODUCCIÓN}

\section{1 bosque de La Primavera, al oeste de Guadalajara, es un en- torno tranquilo y hermoso. La Caldera de Coli, el nombre geológico de La Primavera, es un paisaje de pinos, robles, que- bradas profundas y montes bajos y ondulantes. Esta localidad ofrece}

*Profesor investigador del Colegio de Michoacán († 2011).

Doy gracias a los Drs. Elmo Leon y David Wilcox por revisar el argumento técnico de este texto. El Proyecto Arqueológico Teuchitlán está apoyado por El Colegio de Michoacán, la Secretaría de Cultura del Estado de Jalisco y el Municipio de Teuchitlán. La maestra Acelia García de Weigand, el maestro Rodrigo Esparza y el Dr. Michael Glascock, quienes me han ayudado en una manera importante para el desarrollo de este estudio. La traducción al español es trabajo del Dr. Eduardo Williams. Las sugerencias del Dr. Andy Roth en la formación de las conclusiones fueron importantes. Si hay errores, son del autor. 
una sensación de serenidad, de un mundo aparte de la caótica, contaminada y congestionada mancha urbana a sus pies. La fresca sombra bajo los árboles, los géiseres y manantiales termales, la cautelosa fauna silvestre-venados, coyotes, conejos, pájaros, serpientes y lagartijas-, así como las flores y los manchones de pasto, presentan un contraste tan completo con la vecina ciudad que se vuelve difícil de creer que tal lugar de vida silvestre pueda existir tan cerca. La Primavera realmente es una tierra de contrastes, de muchos usos y de múltiples visiones: para los habitantes urbanos se ha convertido en un refugio cercano; para el naturalista es una oportunidad de examinar la vida silvestre literalmente en la puerta de Guadalajara; para las aldeas vecinas es un lugar para pastar su ganado y obtener leña; para la Comisión Federal de Electricidad (CFE) es un área potencial de energía termoeléctrica; para los constructores es una barrera para la ulterior urbanización; para el hidrólogo, es el área de captación del río Salado y una zona de recarga para el manto acuífero que se acaba rápidamente; para el ecólogo, es una de las últimas zonas naturales de la región y un "pulmón” para la ciudad; para el geólogo, es una oportunidad de estudiar una estructura volcánica recientemente activa; para el arqueólogo, es una región de sitios prehispánicos pequeños y de enormes complejos antiguos de extracción de obsidiana. Para casi todo el mundo es una zona que debe protegerse por su propia belleza y valor intrínseco. Es el parque natural más grande de Guadalajara, aunque esté dañado, abandonado y con frecuencia se olvide en la carrera para construir todavía más infraestructura y más suburbios.

Una gran parte de La Primavera ha sido designada como reserva federal protegida, aunque su cuidado se ha delegado al estado de Jalisco. Pero la reserva todavía no cuenta con el apoyo necesario ni con la infraestructura para realmente conservarla y mantenerla. Cada año, usualmente en abril o mayo, aparece una ráfaga de artículos en los diarios de Guadalajara lamentando la falta de inversión y de conservación para el bosque. Muchos de estos breves textos están bien investigados y bien escritos (un reciente ejemplo es el de Mónica Pérez Taylor 2007), pero parecen tener limitado efecto a largo plazo. Si bien, los incendios forestales son el peligro más obvio para la reserva, la agresiva violación por parte de los constructores, la ex- 
tensa extracción de jal, la tala ilícita y el excesivo pastoreo en algunos sectores, la desgraciada tríada de vandalismo, grafiti y basura, así como el deterioro progresivo general y la degradación, caracterizan a grandes y crecientes sectores de La Primavera. Claramente es un área en decline, un recurso que se acaba cada día y que una vez degradado y saqueado ya no se recuperará.

Sin embargo, en realidad los cerros ondulantes y el bosque de hoy no son sino una delgada capa que cubre una caldera que se está erosionado y un lugar de actividad volcánica muy reciente y violenta. En general, una caldera es fundamentalmente diferente de un estrato-volcán, tanto en su geomorfología como en el tipo de actividad que caracteriza su volcanismo. Los estrato-volcanes parecen verdaderas montańas y en su mayor parte producen erupciones de lava. En Jalisco, los volcanes de Tequila (inactivo, pero no totalmente muerto) y de Colima (entre los más activos del Nuevo Mundo) pertenecen a esta variedad. Otro estrato-volcán cercano y recientemente activo es el de Ceboruco, en Nayarit. Los estrato-volcanes pueden producir eventos piroclásticos ${ }^{1}$ como el Vesubio, cerca de la actual ciudad de Nápoles, que cubrió a las ciudades romanas de Pompeya y Herculano en 79 d.C., y más reciente el monte Santa Helena (Washington). Pero los eventos piroclásticos masivos suceden más frecuentemente con las calderas. Los eventos piroclásticos son explosivos y producen enormes cantidades de tefra, incluyendo ceniza volcánica (jal) de varios tamaños y consistencias. Estos eventos explosivos no producen montańas, sino más bien depresiones en forma de plato rodeadas por los bajos cerros que forman sus bordes u orillas. Esta observación no pretende minimizar el potencial de eventos volcánicos productores de lava; un ejemplo de ello es la for-

1 "Piroclástico" (de la palabra griega "piro" que significa fuego): material de magma y de roca reformada que es expulsado volcánicamente como tefra a temperaturas extremadamente altas y bajo presiones muy altas. "Tefra” es la palabra griega para la ceniza. Actualmente se define como todos los materiales acarreados por el viento que son producidos por erupciones volcánicas. Hay tres tipos básicos de tefra: ceniza (o jal) propiamente, que se define como partículas de menos de $2 \mathrm{~mm}$ de diámetro; lapilli, o escoria volcánica con diámetro de entre 2 y $64 \mathrm{~mm}$; y bombas, que son mayores a $64 \mathrm{~mm}$ de diámetro. El término "silícico" se refiere al principal constituyente de la tefra, fragmentos del elemento sílice o compuestos en los que predomina este material vítreo. 
mación de la Planicie Deccan de la India, que representa uno de los eventos de lava más extensos de la Tierra, que originalmente cubrió un millón de kilómetros cuadrados. ${ }^{2}$

Nuestro interés en el Coli se suscitó a través de nuestros estudios arqueológicos dentro de los valles alrededor del volcán estratificado conocido localmente como volcán de Tequila. Dentro de estos ricos valles se localiza el primer experimento intensivo de un tipo de vida civilizado y complejo en el occidente de Mesoamérica, la tradición Teuchitlán, que durante los periodos Formativo medio y Clásico temprano (800 a.C.-400 d.C.) se desarrolló de una manera dramáticamente diferente de sus vecinos en los valles circundantes, y del resto del occidente de Mesoamérica. Esta civilización temprana estaba orientada hacia las costas del Pacífico, sin recibir influencias importantes ya fuera de los olmecas o de Teotihuacan. Desarrolló un estilo original de arquitectura, que incluye edificios circulares concéntricos de proporciones monumentales, enormes juegos de pelota, tumbas de tiro profundas con varias cámaras y ricas ofrendas, chinampas, un arte elaborado y especializado de figurillas, vasijas de cerámica y joyería de obsidiana, así como otros indicadores de un área económica clave, incluyendo un cierto grado de urbanización y un perfil demográfico elevado (Weigand 1993, 2006; Weigand y Beekman 2008).

Este temprano desarrollo político y económico diferencial dentro de la zona obviamente tuvo una base en los sistemas ecológicos y geomorfológicos de la región. Esta riqueza natural consistió en lo siguiente:

1. Agua (pantanos, lagos, ríos, arroyos y manantiales, la mayoría actualmente secos o muy contaminados);

${ }^{2}$ La Planicie de Deccan, o Traps, está localizada en el centro-oeste de la India y es una de las más grandes provincias ígneas del mundo. La erupción ocurrió hace unos 65 millones de años, al final del Cretácico y originalmente cubrió más de un 1,000,000 km² (de los cuales queda la mitad tras la subsiguiente erosión y deriva continental). Algunos geólogos y paleontólogos piensan que este evento pudo haber contribuido a la extinción de los dinosaurios. El volumen se ha calculado en $512,000 \mathrm{~km}^{3}$, y tiene hasta $2 \mathrm{~km}$ de profundidad en algunas áreas. Fue ocasionado por una gran pluma de manto conocida como el Hotspot Reunión (Nasa 2007). 
2. Suelos (mezclas muy productivas de depósitos aluviales y ceniza volcánica);

3. Un clima benéfico (con patrones de lluvia predecibles y suficientes);

4. Una densa cubierta de bosque (mezcla de pino y roble, actualmente muy deforestada);

5. Obsidiana (para todos tipos de herramientas y de ornamentos).

Este perfil de recursos dio a la Región Valles una ventaja competitiva en el contexto de las sociedades complejas que se desarrollaron dentro del occidente de Mesoamérica. Pocas zonas de tierras altas dentro de Mesoamérica estaban tan bien proveídas. Importantes configuraciones socioculturales siguieron desarrollándose en el área general durante los periodos prehispánicos tardíos. No fue por accidente que el definitivo lugar para Guadalajara fuera escogido en la época colonial en esta región, o que la ciudad contemporánea siga prosperando dentro de esta zona rica con tantos dones de la Naturaleza.

Los últimos episodios de volcanismo fueron los eventos que permitieron el desarrollo de los ricos suelos mixtos. Fue la Caldera de Coli la que produjo esta última capa de depósitos de tefra-sílice-jal en la Región Valles (que se conoce como la toba de Tala) y en la región vecina donde se encuentra actualmente Guadalajara. Además, los eventos volcánicos en Coli produjeron eventualmente una de las más extensas series de afloramientos de obsidiana en el mundo, en tercer lugar después del Valle Rift de África oriental y de la Planicie de Oregon en los Estados Unidos. En cuanto a la calidad, estos depósitos están entre los mejores y de más variado color y textura en el mundo. ${ }^{3}$ El eje sobre el cual aparece la mayor parte de la obsidiana es el eje Tequila-Coli, aproximadamente paralelo a la zona que separa al Bloque de Jalisco del sector de la Sierra Madre Occidental de la placa tectónica de Norteamérica (véase infra), y la orilla sur de la

${ }^{3}$ Estas observaciones comparativas sobre la obsidiana vienen de Michael Glascock y de su proyecto analítico sobre este material en el reactor para la investigación localizado en la Universidad de Missouri en Columbia. Su técnica analítica es la activación de neutrones. Para algunos resultados de su proyecto sobre la obsidiana del eje Tequila-Coli, véase Weigand et al. (2004). 
barranca del Río Grande de Santiago. Los complejos masivos de minería prehispánica caracterizan a este eje. Nuestra evaluación de la minería antigua de obsidiana ha ido avanzando junto con nuestra prospección y documentación de más complejos mineros. Ahora nos estamos enfocando en el área de Coli. Nuestro entendimiento de la contribución de los volcanes a los suelos aluviales dentro de la región nos hizo ver hacia la Caldera de Coli, que es la razón para este breve estudio.

\section{LA FORMACIÓN Y CARACTERÍSTICAS DE LAS CALDERAS}

Las calderas son rasgos geológicos bastante comunes, y muy frecuentemente están entremezclados con otros tipos de volcanes. Algunas calderas se clasifican como "súpervolcanes", que han producido explosiones muy destructivas y cantidades masivas de tefra. El enorme volumen de material expulsado ha alterado dramáticamente los paisajes de cerca y lejos, afectando además el clima global durante años, si no es que décadas. Un "índice de explosividad volcánica" o IEV existe para caracterizar estas explosiones masivas, junto con un "equivalente de rocas densas" o ERD, para calcular la cantidad de material expulsado (Newhall y Self 1982). Igual que la escala utilizada para medir terremotos, cada intervalo de la escala de IEv representa un incremento de 10 puntos en la explosividad. Algunos de estos eventos explosivos son difíciles de imaginar: una caldera con clasificación IEV de 8, por ejemplo, expulsaría un ERD de por lo menos $1,000 \mathrm{~km}^{3}$ de material piroclástico. Hay varios ejemplos de esto: el Lago Taupo (Nueva Zelanda) de hace unos 25,000 años, que expulsó aproximadamente $1,170 \mathrm{~km}^{3}$; el Lago Toba (Sumatra) de hace unos 75,000 años, que expulsó unos 2,800 $\mathrm{km}^{3}$; Yellowstone (Wyoming), de hace unos 2.2 millones de años, que expulsó $2,500 \mathrm{~km}^{3} \mathrm{y}$ de nuevo hace unos 640,000 años, con 1,000 km³ La Garita (Colorado) de hace 27.8 millones de años, que expulsó un ERD de unos $5,000 \mathrm{~km}^{3}$ (Simkin y Siebert 1994; Sigurdsson 1999; Mason et al. 2004; Bindeman 2006). Obviamente, la Caldera de Coli no pertenece a esta categoría de "súper volcanes", que también se llaman "megacolosales" (IEV-8) y súpercolosales (con clasificación de IEV-7 
Figura A. Representación estilizada de los pasos involucrados en la formación de una caldera
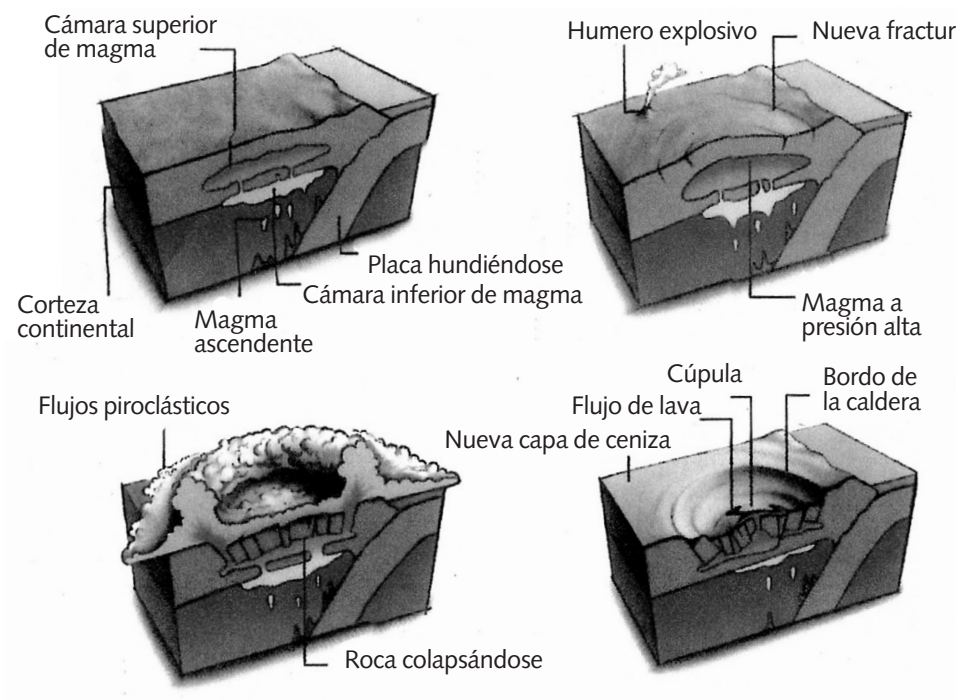

y un ERD de por lo menos $100 \mathrm{~km}^{3}$ ). La Caldera de Coli tiene un ERD de aproximadamente $45 \mathrm{~km}^{3}$ (Mahood 1981), por lo que recibe una clasificación de simplemente "colosal" o IEv-6. ${ }^{4}$ Por lo tanto, no son los índices de IEV o de ERD de Coli los que son tan monumentales, sino más bien la manera en que afectaron a la Región Valles (incluyendo la zona metropolitana general de Guadalajara); esto es lo que nos interesa en este breve estudio.

Como ya se mencionó, las calderas tienen una geomorfología en forma de plato, usualmente circular con una cuenca o valle interior deprimido. Estas depresiones son una característica fundamental de las calderas, ya que en lugar de formarse como eventos que construyen montańas, su formación involucra colapsos. La figura A es una representación estilizada de la manera en que se forman las calderas,

${ }^{4}$ Comparativamente, la última erupción en 1980 del monte Santa Helena, que recibiera tanta publicidad, se clasificó como un evento IEV-5, que produjo un índice de ERD de $1 \mathrm{~km}^{3}$ de material expulsado. 
que sintetiza con claridad lo que sucedió en la Caldera de Coli hace unos 95,000 años. Las calderas usualmente se localizan dentro de áreas de movimiento de placas por actividad tectónica, por lo que al igual que otros tipos de volcanes, se generan cuando una cámara de magma superior penetra al manto de la Tierra. En el caso de una caldera, una cámara baja de magma empieza a ejercer presión sobre la de arriba, formando dentro de ella un sistema de presión alto y expansivo. Entonces empiezan a formarse respiraderos alrededor de las orillas de la cámara superior, los cuales causan fracturas en la corteza superficial; en esta etapa la lava es el producto más común que sale a la superficie a través de los respiraderos, aunque también pueden escapar gas y algunos materiales piroclásticos. $\mathrm{Al}$ aumentar el número y tamaño de los respiraderos, un segmento circular de manto se debilita y eventualmente se colapsa dentro de la cámara de magma superior. El manto colapsado cae dentro del magma súper calentado y presurizado, convirtiéndolo todo en material piroclástico. Esta ocurrencia produce una explosión extremadamente violenta. Esta combinación de manto fundido y magma de la cámara superior ha sido analizada químicamente en varias calderas (Bindeman y Valley 2001; véanse también Knight et al. 1986; Rose y Chesner 1987). Los flujos piroclásticos que resultan de esto son masivos, aunque sólo sea a escala regional; además pueden alcanzar temperaturas de hasta $700^{\circ} \mathrm{C}$, y velocidades de expansión de hasta $400 \mathrm{~km}$ por hora. Nada puede correr más rápido que ellos ni sobrevivir una vez que es alcanzado, como lo atestiguan los cuerpos evaporados de Pompeya y Herculano.

Una vez que pasa el evento piroclástico, la cámara superior se vacía de magma en gran medida, y se forma la distintiva depresión o valle en forma de plato dentro de la caldera, a causa del colapso del manto. El borde de la caldera, compuesto en gran medida de materiales de tefra, rodea a esta depresión interior, usualmente visible como una serie de cerros bajos y conectados o de lomas curvas. $\mathrm{Al}$ empezar a llenarse de nuevo la cámara superior, escapa el gas y con frecuencia se forman géiseres y domos de lava dentro de la caldera o en la orilla interior del borde, oscureciendo un poco la clásica forma de plato. De esta manera, las erupciones subsecuentes son de menor 
escala. Éstas se caracterizan más por lava que por explosiones piroclásticas, al menos hasta que la cámara de magma inferior empieza de nuevo a ejercer presión, reformando y rellenando la cámara superior. $\mathrm{Al}$ reconstruirse ambas cámaras, el ciclo puede repetirse muchas veces, al colapsarse de nuevo la nueva área del manto dentro de la cámara superior. En algunos casos sólo segmentos pequeños del nuevo manto se ven involucrados en los colapsos subsecuentes. Por ejemplo, en Yellowstone un segundo colapso de manto fue considerablemente más chico que el primer evento mayor, aunque generó una masiva erupción de tefra.

Típicamente las calderas se forman ya sea dentro o cerca de zonas de subducción (las áreas donde se encuentran dos o más placas tectónicas y donde una se mueve por encima de otra), o sobre puntos calientes (hot spots) (donde una placa tectónica se mueve sobre una pluma de magma a alta presión). La primer situación caracteriza al gran súper volcán de Toba en Sumatra; la segunda a Yellowstone. Estos dos súpervolcanes, junto con Taupo, forman la clase altamente peligrosa de megacolosales, y sus erupciones tienen el mismo impacto sobre la Tierra que una colisión con un asteroide, afectando al clima durante muchos años y cubriendo una enorme zona con tefra, así se alteran los suelos y la vegetación en enormes áreas.

\section{El lugar del Coli en la geología Regional}

La clave para entender la geología del Coli es el Bloque Jalisco (BJ) y la división entre éste y el sector de la placa tectónica de Norteamérica que corresponde a la Sierra Madre Occidental (sMo). El BJ es un fragmento semidesprendido de esta placa tectónica, metido en el triángulo donde la placa de Norteamérica choca con las de Rivera y Cocos. El BJ, y por tanto el Coli, yacen dentro de lo que se ha llamado correctamente una "juntura triple de grieta continental" (Aguirre 1996: 10; cfr. Ferrari et al. 2000; Wallace et al. 1992; Rosas et al. 1996). Detrás de las placas Rivera y Cocos está la placa del Pacífico (figura B). La orilla norte del BJ está aproximadamente paralela a la barranca del río Grande de Santiago, y ciertamente ubicada dentro 
Figura B. Ubicación del Bloque Jalisco en relación con las placas de Norteamérica, Rivera, Cocos y del Pacífico (de Rossotti et al. 2002, 3)

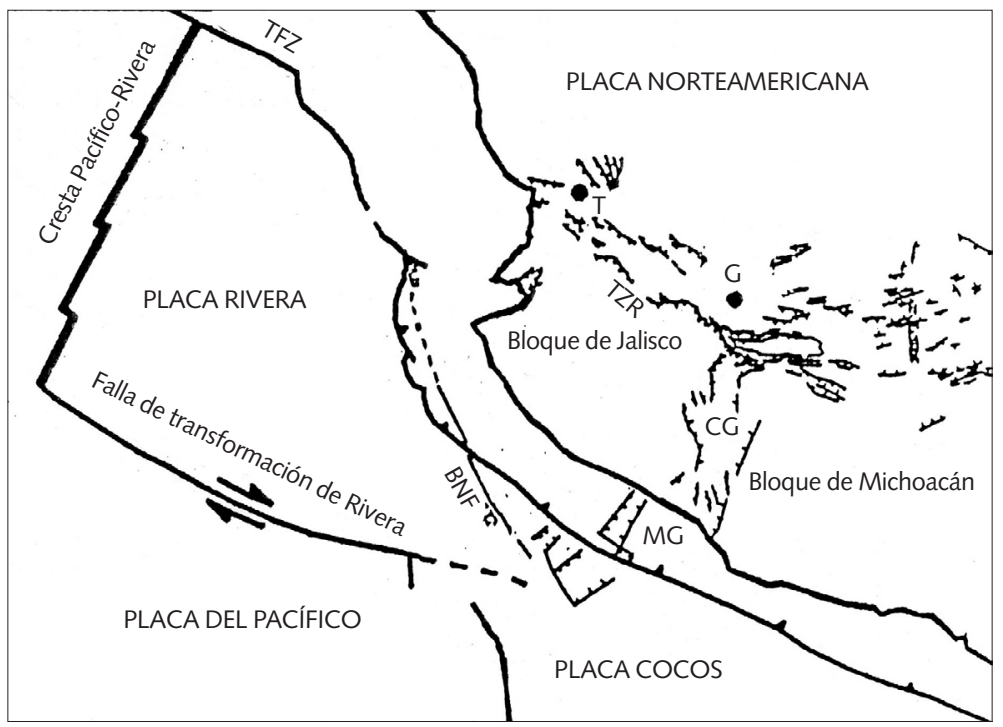

del área enmarcada por este río y el sistema del río Ameca. La ubicación exacta de la Caldera de Coli a lo largo de esta división es algo relativamente seguro. Los estudios de Ferrari et al. (1997, 1999), que ofrecen el más comprehensivo análisis del bloque, sitúan al Coli en la orilla más al sur de la placa de Norteamérica, directamente en los límites de la orilla norte del BJ; Rosotti et al. (2002) coinciden con esta ubicación (figura C).

Ya sea que se localice al norte o al sur de la división entre el BJ y el resto de la placa de Norteamérica, algo importante es que la existencia de la caldera y su actividad están íntimamente relacionadas con ese lindero, y con la inherente inestabilidad que se encuentra ahí. La geología y la geomorfología del Bu difieren un poco de las secciones adyacentes de la placa de Norteamérica, representada localmente por el sector de la SMO (Barrera 2002; Ferrari et al. 1999, 2003; Ferrari 1995; Rossotti et al. 2002). También la orilla sureste del вJ ha sido definida (Garduño et al. 1998), pero no debe importarnos 
Figura C. El Bloque Jalisco y la ubicación de la Caldera de Coli justo sobre la orilla norte del bloque (de Ferrari et al. 1999, 79). Claves: $\mathrm{LP}=$ La Primavera o la Caldera de Coli; Teq=Volcán de Tequila; $\mathrm{CE}=$ Volcán de Ceboruco; SP= Caldera de San Pedro Lagunillas; TE= Tepetiltic; SJ= San Juan

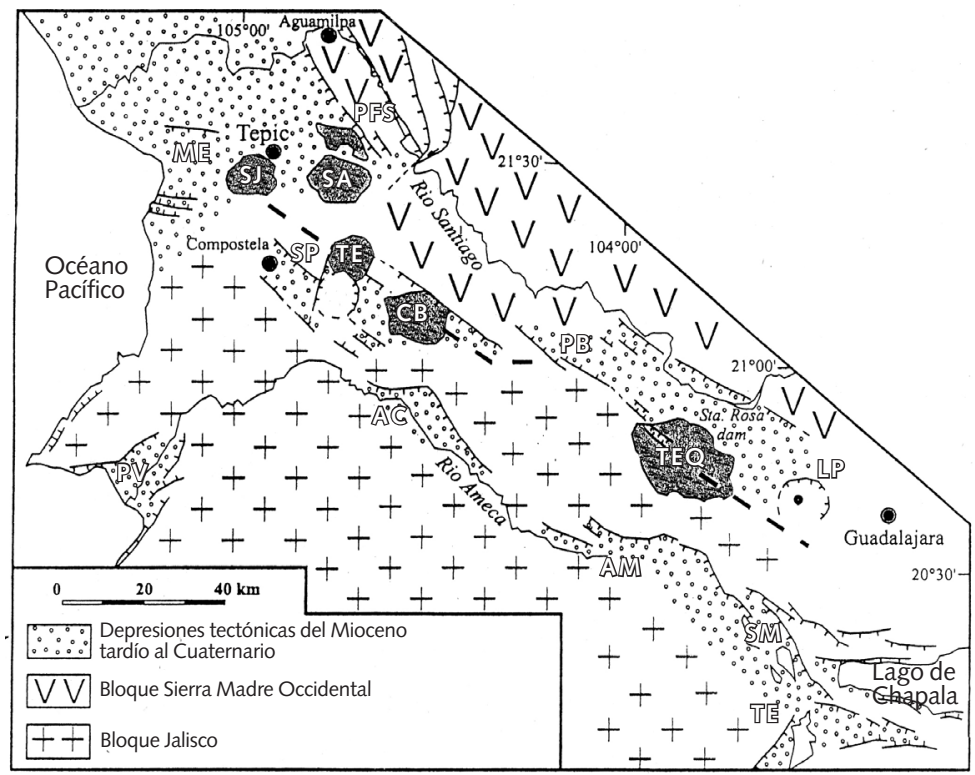

aquí, tampoco la subducción y el agrietamiento activos a lo largo de la orilla oeste de la Franja Transvolcánica Mexicana (FTVM), aunque obviamente estas actividades tectónicas son los principales móviles que afectan (ya sea de manera directa o indirecta) la formación del BJ y los subsiguientes desarrollos entre éste y el sector de la SMO ( $c f r$. Luhr et al. 1985; Allan et al. 1991).

El desarrollo del BJ tiene una historia larga y mal definida, completamente inserta en la relación entre la SMO y la FTVM. La historia geológica observable empieza en tiempos del Mioceno para la SMO. El episodio tardío del Mioceno en la región general de Guadalajara produjo una capa de sucesiones basálticas, que se conoce como grupo del río Santiago. Todavía dentro del Mioceno, un evento o even- 
tos silícicos formaron el grupo de Guadalajara, a los que siguieron otros episodios en el Plioceno temprano, caracterizados por mezclas y fusiones complejas entre magmas silícicas y basalto. Esto a su vez fue seguido en el Plioceno tardío por episodios de formación de domos y flujos riolíticos. El volumen agregado del grupo de Guadalajara es de unos $1,800 \mathrm{~km}^{3}$. El volcanismo silícico cubre aproximadamente $1,000 \mathrm{~km}^{2}$, con un volumen agregado de unos $300 \mathrm{~km}^{3}$. Este volumen aproximado equivale a nueve veces la cantidad expulsada por la Caldera de Coli durante su periodo pleistocénico, con su evento tefrítico que ocurrió, como ya se mencionó, hace unos 95,000 ańos. Rosotti et al. (2002) por lo tanto calculan un ERD de alrededor de $35 \mathrm{~km}^{3}$ para la erupción tefrítica del Coli, aunque Mahood (1981) piensa que un total de $45 \mathrm{~km}^{3}$, que produjo la toba de Tala (véase abajo), es más exacto. Los núcleos de los estudios de la Comisión Federal de Electricidad en el centro del Coli muestran de buena manera no sólo la historia geológica de la caldera, sino también las capas del sector de la smo debajo de ella (figura D). La falta de conexión entre el BJ y la sMo es evidente en las capas inferiores ${ }^{5}$ (Ferrari et al. 1999; Rosotti et al. 2002).

Gail Mahood ha realizado el estudio más detallado de la Caldera de Coli propiamente (Mahood 1980, 1981a, 1981b; Mahood y Drake 1982). Otros estudios hechos por Gutiérrez (1988), Walker et al. (1981) y Yokoyama (1991) complementan y expanden el trabajo de Mahood. Las siguientes observaciones sobre la Caldera de Coli se tomaron principalmente de Mahood, además de considerar los otros trabajos citados arriba, así como de mis propias observaciones en el campo. Una pequeńa cantidad de calderas en el occidente de México también han recibido atención en detalle de los geólogos, por ejemplo, la de Los Humeros (Robin y Nicholas 1978; Negendank et al. 1985) y la de Amealco (Aguirre 1996); este último estudio es muy detallado y bien ilustrado. La caldera de San Pedro Lagunillas (a 15 $\mathrm{km}$ al este-sureste de Compostela, Nayarit) también ha sido estudia-

${ }^{5}$ Este resumen breve y parcial de ninguna manera representa el detalle disponible en los estudios de Ferrari/ Rossotti, y no deberá tomarse como un resumen adecuado de esas investigaciones. 
Figura D. Perfiles de pozos de la Comisión Federal de Electricidad de La Primavera/Caldera de Coli. Las elevaciones en metros están a la izquierda de la ilustración, con las fechas calculadas a la derecha (tomado de Ferrari et al. 1999, 76)

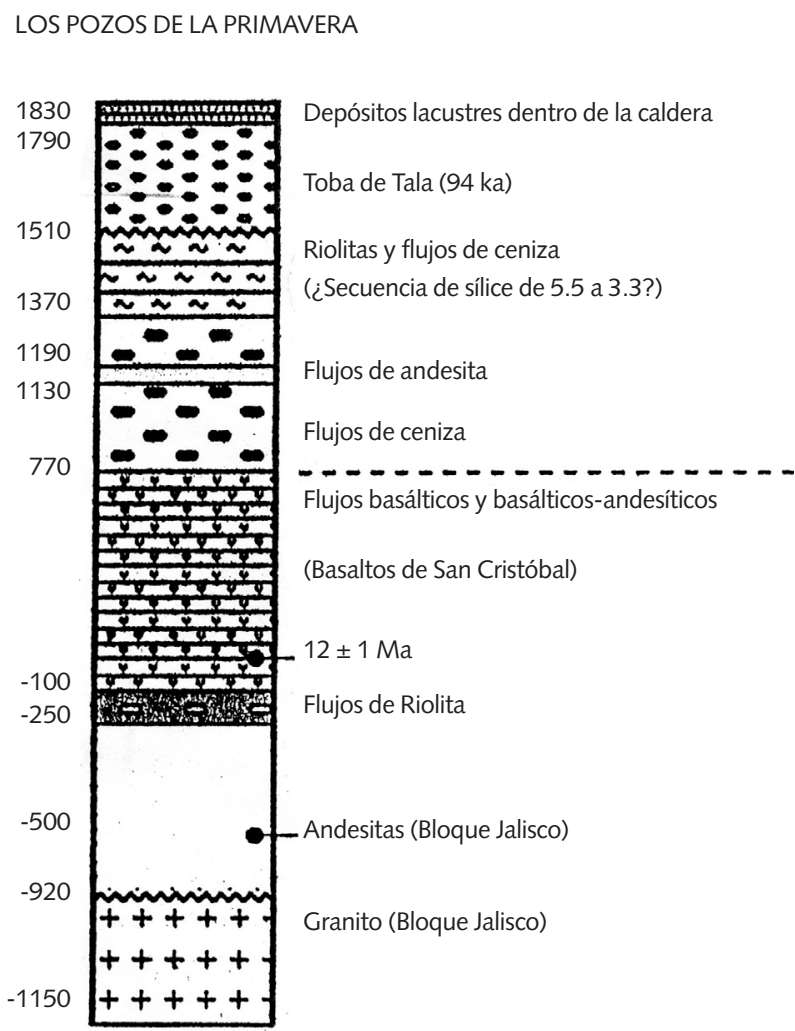

da, con las observaciones más detalladas hechas por Castillo de la Cruz (1992; véase también a Ferrari et al. 2003 para una descripción de la zona general de San Pedro-Ceboruco). En muchos sentidos, es la estructura de caldera "gemela" a la del Coli, bordeando la orilla norte del BJ. La Caldera de San Pedro parece fechar hacia el Pleistoceno medio ( $c a$. 45-50,000 años); es una formación elíptica de aproximadamente 7-10 km de ancho. El borde de la caldera puede 
verse en su lado este. Los flujos de lava de San Pedro tienen fechas de alrededor de 25-28,000 años, aproximadamente en paralelo con la secuencia de eventos observable en la Caldera de Coli (infra). Se siguieron formando domos en San Pedro durante el Holoceno.

Existe otra caldera en la Laguna de Tepetiltic, dentro del campo de volcanes de Tepetiltic (a unos $20 \mathrm{~km}$ al este-noreste de Compostela; Ferrari et al. 2003: mapa en la página 172). Este volcán/ caldera parece tener aproximadamente la misma fecha que San Pedro, aunque Tepetiltic es un volcán poligenético más que estrictamente piroclástico. Las relaciónes entre San Pedro y Tepetiltic todavía no se han determinado (Ferrari et al. 2003; las fechas de estos rasgos geológicos son de Petrone et al. 2001). El estrato-volcán en Ceboruco es enteramente de fecha del Holoceno; la última erupción registrada ocurrió entre 1870 y 1875 . Este volcán también tiene una secuencia de caldera, y se alza a una elevación de 2,000 m, aunque sería $500 \mathrm{~m}$ más alto si no tuviera en la corona la formación de dos calderas concéntricas que se formaron cuando la cámara de magma debajo del Ceboruco se colapsó para formar la caldera exterior (con un diámetro de 4 km, véase a Nelson 1980, 1986). También se han llevado a cabo estudios estructurales dentro del BJ propiamente, por ejemplo el examen de la grieta de Tepic-Zacoalco (GTZ) que subyace al volcán de Tequila y al sector suroeste de la Caldera de Coli (Ferrari y Rosas 1999). El actual curso del río Ameca obedece a una de las tres depresiones de la GTZ, mientras que esta última parece haber sido una respuesta a la deformación dentro de las placas tectónicas del BJ. Este es sólo uno de muchos rasgos dentro del BJ.

Dentro del área general de la Región Valles, según mi propia cuenta, existen 36 conos cineríticos que también produjeron material tefrítico, usualmente lapilli y bombas en lugar de ceniza propiamente. Estos conos varían entre estructuras muy pequeñas y simétricas, como el Cerro Molcajete, cerca de Santa Rosalía (municipio de Etzatlán) y la Caldera de Los Lobos (municipio de Teuchitlán), hasta cerros muy grandes. Ninguno de ellos ha sido fechado, aunque algunos parecen ser bastante recientes por la falta de erosión y su geomorfología bien conservada. Además, algunos tienen escurrimientos de agua caliente y escapes de gas, indicando actividad 
latente debajo de sus estructuras superficiales. Si bien, estos conos han producido grandes cantidades de lapilli y de bombas, no es probable que hayan contribuido de manera importante a los extensos depósitos de ceniza y de jal en la región. Un estudio como el de Hasenaka y Carmichael (1987) en Michoacán y Guanajuato todavía no se ha llevado a cabo en los conos cineríticos de la Región Valles, los cuales junto con sus campos cineríticos representan todavía una región virgen para la exploración geológica.

\section{La Caldera de Coli, per $S E$}

Aunque de forma burdamente circular y con una depresión central en forma de plato todavía visible (pero leve), la morfología general de la Caldera de Coli está deformada y alterada por la erosión y por el subsiguiente volcanismo de bajo nivel. Estas deformaciones y alteraciones han sucedido de manera más bien rápida, dada la juventud geológica de la estructura. Como ya se mencionó, el Coli es un producto del Pleistoceno, aunque todavía debe considerarse como potencialmente activo. Por otra parte, como ya se mencionó, el rango de tiempo sugerido para la actividad de la caldera es de entre 120,000 y 20,000 años, aunque el evento principal, que produjo tanto a la caldera como rasgo geomorfológico y a su resultante toba de Tala, ha sido fechado hacia 95,000 años en el pasado. A continuación se presenta una descripción de la Caldera de Coli y de la secuencia y naturaleza de los eventos que la formaron.

La caldera es relativamente pequeña si la comparamos con Yellowstone o Long Valley (California). En su configuración original la Caldera de Coli era más o menos circular con diámetro de $11 \mathrm{~km}$, y cubría una superficie aproximada de $96 \mathrm{~km}^{2}$. La sección interior es la de forma menos bien definida, a causa de la subsiguiente erupción y elevación de domos (véase abajo), pero medía aproximadamente $6 \mathrm{~km}^{2}$, cubriendo unos $35 \mathrm{~km}^{2}$. Las orillas exteriores de la caldera han sufrido bastante erosión, por lo que el diámetro aparente es un tanto menor en la actualidad. Esta erosión es más visible en las orillas oeste y noroeste, frente a los pueblos de Tala y Huaxtla, respectivamente, por lo que permite que los principales cuerpos de 
agua que quedan en el área salgan desde esa dirección (el Arroyo de las Ánimas y el río Salado, el único arroyo perenne que queda en el área) y a través de rupturas en el borde sur (los arroyos de Agua Caliente y La Villita). Las orillas preservadas más altas de la caldera están en los lados sur y este, enfrente de San Isidro Mazatepec y de Guadalajara respectivamente. El valle interior en forma de plato, como ya se mencionó, está muy deformado a causa de la erosión y del subsiguiente volcanismo piroclástico. Las elevaciones más altas son de más de 2,000 m (llegando hasta 2,270 m), o sea unos 5-600 $\mathrm{m}$ sobre el piso de los valles circundantes. Los cerros prominentes son la Mesa el Nejahuate y el cerro del Capulín en el norte, cerca de La Venta del Astillero; el cerro El Tule en el este, cerca de Santa Ana Tepetitlan; y el Cerro de San Miguel en el sureste, cerca de Tala.

Los afloramientos de obsidiana aparecen en varias áreas dentro de la caldera y su borde: la zona conocida como El Pedernal está en el sector central; el enorme complejo minero prehispánico que tiene algunas minas subterráneas con cámaras está justo al norte de San Isidro Mazatepec; y hay afloramientos con grandes bloques localizados justo al sur del pueblo de La Primavera, aunque también existen otras áreas. Excelentes perfiles de la toba de Tala pueden verse al norte de Cuxpala y al sur de Huaxtla en áreas del borde expuestas por la extracción de jal. Los depósitos profundos de la toba de Tala al norte de la Caldera de Coli dieron a los españoles la oportunidad de excavar una gran serie de qanat (acueductos subterráneos, también llamados "galerías filtrantes"). El qanat de La Venta fue enteramente excavado en la toba de Tala, y tiene un túnel con longitud total de $8 \mathrm{~km}$, por lo que es la más grande estructura de irrigación de este tipo en el Nuevo Mundo (Beekman et al. 1996), aunque hay unas 25 estructuras de menor tamańo en el área general. ${ }^{6}$

\footnotetext{
${ }^{6}$ Un sistema más pequeño de qanat todavía funciona en Guadalajara (en Los Colomos), contribuyendo una parte imitada de la red de agua potable de la ciudad. Un sistema franciscano en Tlaquepaque ha sido cubierto por la urbanización, y ya no funciona. El área de La Venta tiene el sistema más complejo, aunque ninguno ha sido mantenido y con una excepción ninguno proporciona agua en la actualidad. La excepción es La Gotera, un pequeño qanat al este de La Venta. El agua que era filtrada gradualmente dentro de estas estructuras proporcionaba este recurso de manera confiable todo el ańo. El agua de los qanats es excepcionalmente limpia, por lo que sirvió como fuente para tomar y dar al
} 
El evento que produjo la caldera también expulsó el ERD de 45 $\mathrm{km}^{3}$ llamado toba de Tala, ${ }^{7}$ tan evidente en los depósitos por toda la Región Valles, incluyendo la zona de Guadalajara propiamente. El grosor de la capa de toba de Tala varía considerablemente por toda el área, y dentro de la misma caldera los pozos de la CFE muestran una profundidad de $280 \mathrm{~m}$ (figura D). A lo largo de las orillas este y exterior de la caldera, la ya mencionada extracción de jal ha expuesto algunos perfiles de por lo menos esas mismas dimensiones. Los depósitos en las zonas que yo he examinado son casi completamente uniformes, sin mostrar varvas u otras zonas internas. En general, el depósito se caracteriza por un color gris claro o medio, muy finas texturas sujetas a desintegración en partículas de polvo fino, y en relativamente inestables caras una vez que se excavan los perfiles. Los perfiles expuestos rápidamente se intemperizan hasta adquirir un color entre gris oscuro y café gris, siguiendo todavía relativamente inestables. Si se inunda por periodos grandes, la toba de Tala puede adquirir un tono amarillento. Mahood (1981) señaló zonas químicas entre los primeros materiales de erupción y la última parte metaluminosa. ${ }^{8} \mathrm{Si}$ bien esta zona es químicamente perceptible, como ya se dijo, no se puede reconocer visualmente. Según el estudio de Mahood de 1981, el asentamiento de los cristales y el creciente fundido parcial no pudieron haber producido la gradación química del depósito de la toba de Tala. Parece que esta variabilidad se debe al transporte de metales traza como complejos volátiles dentro de la gradiente termal y gravitacional en el magma no saturado

ganado, así como para la irrigación. Los sistemas de qanat alrededor de Guadalajara hicieron una contribución crucial a la economía colonial del área, especialmente para la producción del trigo de invierno.

${ }^{7}$ En su estudio de 1980, Mahood calcula un ERD de $20 \mathrm{~km}^{3}$ para el evento que produjo la toba de Tala. La cifra de $45 \mathrm{~km}^{3}$ viene de su estudio de 1981. De cualquier manera, este fue el último evento eruptivo importante que produjo tefra en el área general, por lo que es responsable de las mezclas de suelo a las que hemos aludido en este texto.

${ }^{8}$ El estudio químico de Mahood (1981) distinguió dos zonas principales en la toba de Tala. Los primeros materiales expulsados son ricos en los siguientes elementos: $\mathrm{Na}$, Rb, Cs, Cl, F, Zn, Y, Zr, Hf, Ta, Nb, Sb, HREE, Pb, Th y U. La última parte metaluminosa es rica en: K, LREE, Sc y Ti. Varios elementos (Al, Ca, Mg, Mn, Fe y Eu) son consistentes dentro del rango del error analítico. Véase a Noble et al. (1974) para un tipo relacionado de análisis químico para las tobas del valle de Eureka (California). 
en agua. La secuencia de eventos que produjeron la caldera, como fue reconstruida por Mahood, es la siguiente:

1. El primer evento fue hace unos 120,000 años;

2. El evento explosivo que produjo toda la toba de Tala ocurrió hace unos 95,000 años;

3. Casi contemporánea (pero un poco después) con ese evento (2) fue la formación del primer domo posterior a la caldera dentro de la estructura en la porción sur-centro;

4. Justo después de que se formó ese domo, otro hizo erupción en el área norte-centro;

5. Antes del momento de la erupción de los dos domos centrales, un gran lago se había formado en la depresión dejada por el colapso del manto dentro de la cámara de magma superior;

6. Estos dos domos centrales hicieron erupción dentro de este lago, perturbando un poco la formación de sedimentación aluvial, aunque dejaron un gran horizonte de pómez como marcador estratigráfico (figura D);

7. Después de la formación de ese horizonte, varios domos anulares hicieron erupción, uno sobre el segmento noreste de la zona de fractura original, mientras que el otro cruzaba el lago. Todos estos eventos posteriores a 120,000 ańos, desde la erupción explosiva que produjo a la toba de Tala (hace 95,000 años), la formación del lago y las dos erupciones del domo central, hasta los domos en forma de anillo, ocurrieron dentro de un periodo de 5-10,000 años. En ese periodo -tan breve en términos geológicos- se formó rápidamente la forma básica de la caldera como la vemos actualmente;

8. El lago, aunque alterado, siguió existiendo hasta hace unos 75,000 ańos, cuando nuevos domos en forma de anillo hicieron erupción a lo largo del margen sur del lago;

9. Hubo un levantamiento a causa de la oleada de nuevo magma dentro de la cámara superior, y el lago entonces de apareció;

10. Siguieron más erupciones de lava hace unos 60,000 años;

11. Los últimos eventos de lava tuvieron lugar hace unos 20-30,000 años. 
Estos últimos eventos de lava representan sucesivas erupciones de la cámara superior de magma que intentaba rellenarse. Ya que no pudieron establecerse de nuevo los altos perfiles de presión, no existió un nuevo evento piroclástico. Los actuales respiraderos de vapor y manantiales termales están relacionados con las fallas que atraviesan la caldera, causadas por el resurgimiento de magma dentro de la cámara superior. Algunos de estos manantiales tienen temperaturas superficiales promedio de $65^{\circ} \mathrm{C}$, aunque las temperaturas de equilibrio se calculan en $170^{\circ} \mathrm{C}$, encontrándose hasta $270^{\circ} \mathrm{C}$ en los pozos de exploración ( $c f r$. Gutiérrez 1991). Es de esperarse que estos respiraderos sean producidos por sistemas geotérmicos poco profundos, más que por la magma subyacente, como la prospección sísmica parece documentar," aunque esto sigue siendo especulación. Todavía no sabemos hasta qué punto la cámara superior de magma ha sido agotada, o si se está formando de nuevo.

Los materiales de tefra del Coli afectaron a una región circundante bastante grande. Como ya se mencionó, algo benéfico ha sido que este material se mezcló con los suelos aluviales de la región para formar las combinaciones altamente fértiles que existen en la actualidad. La respuesta paleoecológica regional a la deposición de la tefra nunca se ha estudiado en detalle, excepto para señalar la fertilidad del suelo, sus características de buen drenaje, y su general productividad agrícola. La Región Valles tuvo numerosos sistemas de lagos y pantanos grandes durante los periodos Pleistoceno y Holoceno: la Laguna de Magdalena (que fue secada finalmente a mediados del siglo xx); la Laguna Colorada (seriamente contaminada); la Ciénega de San Marcos (drenada); la Lagunita de Palo Verde (que está siendo drenada); las Ciénegas de Teuchitlán (ahora son parte de una presa); Los Lobos y Los Patos (drenados); la Ciénega de Ameca (desecada); las lagunas de San Marcos, de Zacoalco, de Santa Catarina, de Atoyacl Sayula (cuencas salitrosas que se llenan parcialmente de agua en cada estación de lluvias); la Laguna de Zapotlán (contaminada), etcétera.

${ }^{9}$ Mahood (1980) reporta que la prospección sísmica muestra la atenuación de las ondas $\mathrm{S}$ y $\mathrm{P}$, aunque las $\mathrm{P}$ están atenuadas más severamente que las s. La mayor cantidad de atenuación ocurre dentro del área de respiraderos de vapor. 
Claramente, la erupción piroclástica del Coli y la deposición de la tefra dentro del entorno lacustre tuvieron que haber sido importantes para su equilibrio ecológico. Obviamente, los lagos y pantanos se recuperaron lo suficiente durante el Pleistoceno tardío y Holoceno temprano como para ofrecer medios ambientes ventajosos para la megafauna, cuyos restos fosilizados son abundantes en toda la zona. Todavía no se lleva a cabo dentro de la Región Valles un estudio como el de Telford et al. (2004) que examinó los depósitos de tefra en tres lagos dentro del campo volcánico de MichoacánGuanajuato (Zirahuén, Pátzcuaro y Zacapu). Con excepción del estudio ya mencionado de Ferrari y Rosas (1999), que empleó algo de prospección paleo magnética, hasta ahora no se han realizado estudios gravimétricos o magnetotelúricos realmente detallados (como los de Arzate et al. 2006, para el Valle de Banderas, Jalisco/ Nayarit) en las zonas lacustres. Estas prospecciones pueden detectar con mucho mayor detalle las fallas debajo de las profundas estructuras aluviales, las cuales en el caso de la Región Valles podrían estar de alguna manera relacionadas a la evolución y el carácter del Coli. Aunque el BJ tiene muchas características de una micro placa en proceso de separación de la SMO, la mayor parte de su deformación interna parece relacionarse con la actividad dentro de la placa. Esto apoya la idea de una mayor actividad tectónica en el futuro, más que de estabilidad para la Región Valles, la región de Guadalajara y la Caldera de Coli. Este paisaje general todavía está en formación activa, por lo que dista mucho de ser estable.

\section{OBSERVACIONES FINALES}

Aunque este estudio claramente no es un ejercicio técnico de geología profesional, esperamos que al presentar el material sobre la Caldera de Coli en lenguaje accesible, y en español, un público más amplio pueda darse cuenta de la naturaleza e historia de Coli. Históricamente, la Caldera de Coli ha tenido un papel importante en la evolución del presente paisaje, especialmente con la formación de los perfiles de suelo extremadamente ricos de la región y las fuentes y capacidades de agua en la superficie. Los primeros colonos espańoles, tras varios 
intentos fallidos de ubicar a Guadalajara, ciertamente pronto se dieron cuenta del alto valor agrícola de las tierras de las que eventualmente se apoderaron. Ellos descubrieron una canasta de pan natural, apropiada para la producción de cosechas de invierno, especialmente trigo, al igual que maíz y productos de jardines y huertas (Van Young 1981; d'Arc 1973). Con la ya mencionada tecnología del qanat, combinada con la abundante lluvia de verano (tanto para las cosechas de la estación como para recargar el nivel de agua subterránea), florecieron el área inmediata alrededor de Guadalajara, y especialmente la Región Valles. Esta última todavía produce un gran porcentaje del maíz de México. Es una lástima que gran parte del valle de Guadalajara está siendo urbanizado, sacrificando estos excelentes suelos para construir casas y calles. Esa es una pérdida irreparable.

La Región Valles ahora se está anunciando como "paisaje agavero", incluso ha recibido una designación de paisaje cultural por la unESCO, lo cual supuestamente protege los bosques, recursos arqueológicos y sitios históricos. Sin embargo, en términos históricos el actual nivel de producción de agave, que obedece al nuevo mercado internacional para el tequila, es extremadamente reciente. El cultivo industrializado del agave con alto uso de sustancias químicas, está destruyendo rápidamente los suelos de tierras altas. Además, los pesados tractores destruyen lo sitios arqueológicos minutos después de su llegada. La nivelación de la tierra para el cultivo del agave ha destruido hasta los sitios protegidos por el Instituto Nacional de Antropología e Historia. La completa destrucción del complejo del Formativo tardío en Huitzilapa, municipio de Magdalena, perpetrada por la Compañía Herradura, es el caso que ha recibido más publicidad. El paisaje agrícola real fue y sigue siendo el de producción de maíz (Ojeda et al. 2008). El reciente crecimiento del cultivo del agave puede verse en el aumento en la destilación de tequila: 42,000 litros en 1902, 23 millones de litros en 1973, 169 millones en 1998 (Ibid.). El uso irracional de los suelos de tierras altas está propiciando su conversión a depósitos de laterita. Esta laterización ${ }^{10}$ una vez ini-

${ }^{10}$ La laterización ocurre más frecuentemente en suelos que han evolucionado dentro de áreas boscosas que tienen una marcada diferencia entre estaciones de lluvia y de secas. Es un proceso de conversión en el cual los suelos pierden sus contenidos orgánicos y 
ciada destruye rápidamente la fertilidad de los suelos, una preocupación que los agricultores comerciales están ignorando.

De igual manera, los suelos del fondo del valle de la Región Valles también están siendo sacrificados. El uso de estos suelos para la caña de azúcar es una mala estrategia económica para Jalisco, y para Guadalajara en particular. La Región Valles debería ser la canasta de pan de Guadalajara, en vez de importar el maíz de lejos. Esto es especialmente cierto porque la caña de azúcar es antieconómica y la infraestructura para refinarla tecnológicamente atrasada, por lo que requiere de subsidios federales para mantenerla apenas a flote. Aunque los suelos de la Región Valles están siendo maltratados (con la alta aplicación de químicos como fertilizantes, pesticidas, funguicidas y herbicidas), probablemente podrían recuperarse y soportar un programa de agricultura más útil, económicamente posible y sustentable. El agua necesaria para irrigar a la caña de azúcar ahora se está extrayendo con bombas de las reservas subterráneas que son posibles por las gruesas capas de toba de Tala. La caña de azúcar necesita más agua que otras cosechas, y estas reservas de agua se están bombeando más rápidamente de lo que pueden reponerse por la recarga natural. Cada año se secan los pozos más viejos, y cada año la única solución ha sido perforar pozos cada vez más profundos. Solamente cuando la cańa de azúcar se elimine como cultígeno de la región y Guadalajara implemente programas de recuperación, de reciclaje y de uso sustentable del agua, será el regalo del Coli utilizado de manera racional. Los suelos y las aguas subterráneas son esenciales para la vida urbana, una observación tan obvia que no debería ser necesario repetirla. Sin embargo, el constante mal uso de ambos, junto con los cambios climáticos en el nivel global que estamos experimentando, requiere de un cambio en la estrategia para su utili-

microbiológicos, y donde el contenido de sílice está degradado. Los suelos se endurecen (de ahí la palabra latir, que significa "ladrillo" en latín) con altas concentraciones de fosfatos de hierro, y pierden su capacidad de retener el agua de la lluvia. Este proceso es complicado, y se acelera por la alta aplicación de químicos. Una vez iniciado el proceso rápidamente se vuelve irreversible. Los suelos laterizados tardan siglos en recuperar su fertilidad. Véase a Batten et al. (1977) y a Gilluly et al. (1968) para descripciones más detalladas de los procesos de laterización de los suelos. 
zación. La riqueza agrícola de toda la zona es resultado directo de la deposición por el Coli de las tobas de Tala. Al irse erosionando a través de miles de años, se han vuelto altamente productivas, y lo siguen siendo. Actualmente, el gran regalo del Coli a la sociedad humana está siendo mal usado y desperdiciado.

Sin embargo, vivir en la orilla de una caldera semiactiva, sin importar qué tan bajo nivel, aparentemente, sea su actividad, requiere de conocimiento sobre el carácter de este tipo de volcanismo. La menor muestra de actividad renovada o incrementada dentro de la caldera o cerca de ella debería tomarse con la mayor sinceridad. Hay signos de advertencia que podrían permitir que se tomaran las precauciones adecuadas, incluyendo los siguientes:

1. Un incremento en la actividad de los respiraderos;

2. Erupciones de lava o de tefra (sin importar lo pequeño que sean);

3. Temblores, aunque sean menores, dentro o cerca de la caldera.

Si la caldera sufre otro evento piroclástico, los resultados podrían ser enormemente importantes, hasta catastróficos, para Guadalajara y la Región Valles.

\section{REFERENCIAS CITADAS}

Aguirre Díaz, Geraldo J., "Volcanic stratigraphy of the Amealco caldera and vicinity, Central Mexican Volcanic Belt", Revista Mexicana de Ciencias Geológicas, vol. 13, núm. 1, 1996, 10-51.

Allan, J.F., S.A. Nelson, J.F Luhr, I.S.E. Carmichael, M. WoPAT y P.J. WALlaCE, "Pliocene-Recent rifting in SW Mexico and associated volcanism”, en J.P. Dauphin y B.R.T. Simoneit, eds., The Gulf and Peninsular Province of the Californias, American Association of Petroleum Geologists, Memoir 47, 1991, 425. Arzate, Jorge A., Roman Álvarez, Vsevolod Yutsis, Jesús Pacheco y Héctor Lopez Loera, "Geophysical modeling of Valle de Banderas graben and its structural relation to Bahía de Banderas, México", Revista Mexicana de Ciencias Geológicas, vol. 23, núm. 2, 2006, 184-198. 
Barrera, Rosier Omar, "Consideraciones geomorfologías sobre la Sierra Madre Occidental en el norte de Jalisco, México”, Boletín del Instituto de Geografía, unAM, núm. 48, 2002, 44-75.

Batten, James y Sullivan Gibson, Soils: Their nature, classes, distribution, uses, and care, University of Alabama Press, Tuscaloosa, 1977.

Beekman, Christopher, Phil C. Weigand y John J. Pint, "El qanat de La Venta: sistemas hidráulicos de la época colonial en el centro de Jalisco", Relaciones, núms. 63 y 64, 1996, 139-185.

Bindeman, Ilya B., "The secrets of supervolcanoes", Scientific American, vol. 294, núm. 6, 2006, 35-43.

Bindeman, Ilya B. y John W. Valley, "Low ç18-0 rhyolites from Yellowstone: Magnetic evolution based on analysis of zircons and individual phenocryts", Journal of Petrology, vol. 42, 2001, 1491-1517.

Castillo de la Cruz, D., "Reconocimiento estructural y volcanológico en la zona termal del San Pedro Lagunillas, Nayarit, México", Geofisica Internacional, vol. 31, 1992, 407-415.

D’Arc, Hélène, Guadalajara y su región, El Colegio de México, México, 1973.

FERrARI, Lucca, "Miocene shearing along the northern boundary of the Jalisco block and the mouth of the southern Gulf of Mexico", Geology, vol. 23, 1995, 751-754.

Ferrari, Lucca y José Rosas Elguera, "Late Miocene to Quanternary extension at the northern boundary of the Jalisco block, western Mexico: the Tepic-Zacoaclco rift revised", en H. Delgado G., G. Aguirre D., y J.M. Stock, eds., Cenozoic tectonics and volcanism of Mexico, Geological Society of America, special paper \#334, 1999, 1-23.

Ferrari, Lucca y Saul Venegas S., "Tectonics and volcanism of the western Mexican Volcanic Belt”, en Geraldo Aguirre D., J. Aranda Gomez, G. Carrasco Nuñez y Lucca Ferrari, eds., Magmatism and tectonics in central and northwestern Mexico, Instituto de Geologia, UNAM, 1997, 85-129.

Ferrari, Lucca, Giorgio Pasquare, Saul Venegas Salgado y Francisco Romero Rios, "Geology of the western Mexican Volcanic Belt and adjacent Sierra Madre Occidental and Jalisco Block", 
en: H. Delgado Granados, Geraldo Aguirre Díaz, y J.M Stock, eds., Cenozoic Tectonics and Volcanism of Mexico, Geological Society of America Special Paper 334, Boulder, 1999, 65-83.

Ferrari, Lucca, Chiara Petrone, y Lorilla Francalanci, "Generation of oceanic-island basalt-type volcanism in the western Trans-Mexican volcanic belt by slab rollback...", Geology, vol. 29, 2001, 507-510.

Ferrari, Lucca, Chiara M. Petrone, Lorella Francalanci, Takahiro Tagami, Magihiko Eguchi, Pedro Manetti y Sandro Conticelli, "Geology of the San Pedro-Ceboruco graben, western Trans-Mexican Volcanic Belt", Revista Mexicana de Ciencias Geológicas, vol. 20, núm. 3, 2003, 165-181.

Garduño Monroy, Víctor H., Ricardo Saucedo Girón, Zenón Jiménez, Juan Carlos Gavilanes Ruiz, Abel Cortes Cortes y Rosa Maria Uribe Cifuentes, "La falla Tamazula: límite suroriental del Bloque Jalisco y sus relaciones con el complejo volcánico de Colima, México", Revista Mexicana de Ciencias Geológicas, vol. 15, núm. 2, 1998, 132-144.

Gilully, James, Aaron Waters y A.O. Woodford, Principles of Geology, San Francisco, Freeman and Company, 1968.

Gutiérrez Negrin, Luis, "La Primavera, Jalisco, Mexico: geothermal field", Transactions of the Geothermal Research Council, vol. $12,1988,161-165$.

, "Recursos geotérmicos de La Primavera, Jalisco", Ciencia y Desarrollo, vol. 16, núm. 96, 1991, 57-69.

Hasenaka, T. e Ian S.E. Carmichael, "The cinder cones of Michoacan and Guanajuato, central Mexico: petrology and chemistry”, Journal of Petrology, vol. 28, 1987, 241-269.

Knight, M.D., G.P.L. Walker, B.B. Ellwood y J.F. Diehl, "Stratigraphy, paleomagnetism, and magnetic fabric of the Toba Tuffs: constraints on their sources and eruptive styles" Journal of Geophysical Research, vol. 91, 1986, 10,355-10,383.

Luhr, James F., Stephen A. Nelson, James Allan e Ian S.E. CarMICHAEL, "Active rifting in southwestern Mexico: manifestations of incipient eastward spreading-ridge jump", Journal of the Geological Society of America, 1985, 54-57. 
MaHood, Gail A., "Geological evolution of a Pleistocene rhyolitic center-Sierra La Primavera, Jalisco, Mexico", Journal of Volcanology and Geothermal Research, vol. 8, núm. 2-4, 1980, 199230.

"Chemical evolution of a Pleistocene rhyolitic center: Sierra La Primavera, Jalisco, Mexico", Contributions to Minerology and Petrology, vol. 77, núm. 2, 1981a, 129-149.

"A summary of the geology and petrology of the Sierra La Primavera, Jalisco, Mexico", Journal of Geophysical Research, vol. 86, núm. 10, 1981b, 137-152.

Mahood, Gail A. y R.E. Drake, "K-Ar dating young rhyolitic rocks: a case study of the Sierra La Primavera, Jalisco, Mexico", Geological Society of America, Bulletin 93, 1982, 1232-1241.

Mason, Ben G., David M. Pyle y Clive Oppenheimer, "The size and frequency of the largest explosive eruptions on Earth", Bulletin of Volcanology, vol. 66, núm. 8, 2004, 735-748.

NASA (National Aeronautics and Space Administration), "The Deccan Plateau", en Geomorphology from Space, capítulo 3, lámina V-23 ff., Washington, 2007.

Negendank, J.F.W., R. Emmermann, R. Krawczyk, F. Mooser, H. Toвschall y D. Werle, "Geological and geochemical investigations on the eastern Trans-Mexican Volcanic Belt", Geofisica Internacional, vol. 24, 1985, 477-575.

Nelson, S. A., "Geology and petrology of the Volcán Ceboruco, Nayarit, Mexico Pt. 2", Geological Society of America Bulletin, vol. 91, 1980, 2,290-2,431.

, "Geología del Volcán Ceboruco, Nayarit, México, con una estimación de riesgos de erupciones futuras", Revista del Instituto de Geología, vol. 6, unAM, 1986, 243-258.

Newhall, Christopher G. y Steve Self, "The Volcanic Explosivity Index", Journal of Geophysical Research, vol. 87, 1982, 12311238.

Noble, Donal, David Siemmons, Marjorie Korringa, Willam Dickinson, Yehya AL-Rawi y Edwin McKee, "Eureka Valley Tuff, East-Central California and Adjacent Nevada", Geology, vol. 2, 1974, 139-142. 
Ojeda Gastelúm, Samuel, Bruce Bentz y Lorenza López Mestas, "La tradición Teuchitlán, el maguey y el tequila: un debate acerca de su configuración espacial, histórica y cultural: ¿integración o destrucción?”, en: P.C. Weigand y C.S. Beekman, eds., La tradición Teuchitlán: excavaciones en Los Guachimontones, Llano Grande y Navajas, Zamora, El Colegio de Michoacán, 2008.

Pérez TAYlor, Mónica, La Primavera por siempre”, Mural, 7-rv-07, Guadalajara, 2007, 5.

Petrone, C.M., L. Francalanci, R.W. Carlson. L. Ferrari y S. Conticelli, "Volcanic systems in the San Pedro-Ceboruco graben (Nayarit, Mexico) in the light of new K-Ar geochronological data”, Geochemical Journal, vol. 35, 2001, 77-88.

Robin, Claude y Emmanuel Nicolas, "Particularites geochimiques des suites andesitiques de la zona orientale de l'axe transmexicain, dans leur contexte tectonique", Bulletin de la Societe Geologique de France, 7e serie, vol. 20, 1978, 193-202.

Rosas Elguera, José, Lucca Ferrari, Victor H. Garduño y Jaime Urrutia Fucugauchi, "Continental boundaries of the Jalisco block and their influence in the Pliocene kinematics of western Mexico", Geology, vol. 24, 1996, 921-924.

Rose, W.I. y C.A. Chesner, "Dispersal of ash in the great Toga eruption, 75 ka”, Geology, vol. 15, 1987, 913-917.

Rossotti, Andrea, Lucca Ferrari, Margarita López Martínez y José Rosas Elguera, "Geology of the boundary between the Sierra Madre Occidental and the Trans-Mexican Volcanic Belt in the Guadalajara region, western Mexico", Revista Mexicana de Ciencias Geológicas, vol. 19, núm. 1, 2000, 1-15.

Sigurdsson, Haraldar, ed., Encyclopedia of volcanoes, Nueva York, Academic Press, 1999.

Simkin, T. y L. Siebert, Volcanoes of the world, Geoscience Press, Tucson, 1994.

Telford, Richard J., Philip Barker, Sarah Metcalfe y Anthony Newton, "Lacustrine responses to tephra deposition: examples from Mexico", Quaternary Science Reviews, vol. 23, 2004, 23372353.

VAn Young, Eric, Hacienda and Market in Eighteenth-Century 
Mexico: the rural economy of the Guadalajara region, 1675-1820, Berkeley, University of California Press, 1981.

Walker, G.P.L., J.V. Wright, B.J. Clough y B. Booth, "Pyroclastic geology of the rhyolitic volcano of La Primavera, Mexico", Geolog. Rundschau, 70, 1981, 1100-1118.

Wallace, Paul, Ian S.E. Carmichael, Kevin Righter y Tim A. BeCKer, "Volcanism and tectonism in western Mexico...", Geology, vol. 20, 1992, 625-628.

WeIgand, Phil C., Evolución de una civilización prehispánica: arqueología de Jalisco, Nayarity Zacatecas, Zamora, El Colegio de Michoacán, 1993.

, "La tradición Teuchitlán del Occidente de México", en Richard Townsend, ed., Perspectivas del antiguo Occidente de México, Guadalajara, Secretaría de Cultura del Estado de Jalisco, Art Institute of Chicago y Guachimontones y Naturaleza, S.A., 2006, 38-55.

Weigand, Phil C. y Christopher Beekman, eds., La tradición de Teuchitlan: excavaciones en los Guachimontones, Llano Grande y Navajas, Zamora, El Colegio de Michoacán, 2008.

Weigand, Phil C., Acelia Garcia y Michael Glascock, "La explotación de los yacimientos de obsidiana del centro-oeste de Jalisco", en Eduardo Williams, ed., Bienes estratégicos del antiguo Occidente de México, Zamora, El Colegio de Michoacán, 2004, 113-135.

Yokoyama, I. y M. Mena, "Structure of La Primavera caldera, Jalisco, México, deduced from gravity anomalies and drilling results", Journal of Volcanic and Geothermal Research, vol. 47, 1991, 183-193.

FeCha de RECEPCión del aRTículo: 18 de junio de 2007

FECHA DE ACEPTACIÓN Y RECEPCIÓN DE LA VERSIÓN FINAL: 14 de enero de 2011 